\title{
Travail et technique chez les grecs. L'approche de
} J.-P. Vernant

Work and technics in Ancient Greece; J.-P. Vernet's approach

\section{Charles Kanelopoulos}

\section{(2) OpenEdition \\ 1 Journals}

Édition électronique

URL : https://journals.openedition.org/tc/5006

DOI : $10.4000 /$ tc. 5006

ISSN : 1952-420X

Éditeur

Éditions de l'EHESS

\section{Édition imprimée}

Date de publication : 30 juin 2010

Pagination : 335-353

ISSN : 0248-6016

\section{Référence électronique}

Charles Kanelopoulos, «Travail et technique chez les grecs. L'approche de J.-P. Vernant », Techniques \& Culture [En ligne], 54-55 | 2010, mis en ligne le 30 janvier 2013, consulté le 29 septembre 2022. URL : http://journals.openedition.org/tc/5006 ; DOI : https://doi.org/10.4000/tc.5006 


\section{TRAVAIL ET TECHNIQUE CHEZ LES GRECS}

\section{L'approche de J.-P. Vernant}

in Techniques E culture 19, 1992 : 139-160

L'approche anthropologique a été pendant longtemps considérée comme inadéquate pour l'étude du monde grec antique. Pour les orientations dominantes la Grèce ancienne nous est très proche. Patrie de l'humanité européenne, la Grèce parle à travers notre langage, même au moment où nous le contrôlons le plus, et nos problématiques les plus actuelles s'enracinent sur son sol, qui a produit la pensée rationnelle. Le privilège grec ne manque pas d'imprégner les anthropologues eux-mêmes. Ceux-ci, dans leur grande majorité, endossent sans autre forme de procès le préjugé selon lequel leur discipline n’a rien à faire avec le monde classique, le monde de la raison triomphante.

Il a fallu un grand courage à des hommes comme L. Gernet et I. Meyerson pour « relativiser », chacun à sa façon, le cas grec et l'aborder dans son particularisme. C'est aux efforts de ces hommes que se rattachent les recherches de Jean-Pierre Vernant*. Dans sa préface à l'Anthropologie de la Grèce antique de L. Gernet, J.-P. Vernant ne rend pas seulement hommage à son maître mais parle aussi de lui-même et de sa démarche :

« il [Gernet] était chez lui en Grèce ancienne, à la façon d'un ethnologue qui, parti dès l'âge d'homme explorer une terre lointaine, ne l'aurait jamais plus quittée et en comprendrait le peuple du dedans et du dehors, avec le double regard de l'indigène et de l'étranger » (in Gernet 1968a: 11) ${ }^{1}$.

Cette attitude d'« ethnologue » s'entrelace avec la psychologie historique élaborée par I. Meyerson : les fonctions psychologiques changent et ces changements se rendent visibles « à travers l'analyse des œuvres que l'homme a continûment créées et transformées » (Vernant 1960 : 445). En conséquence de quoi il ne peut y avoir de catégories éternelles 
à travers lesquelles on pense "le monde ", les hommes et leurs activités. L'immense curiosité de J.-P. Vernant favorisera par la suite d'autres rencontres : G. Dumezil, C. LéviStrauss, E. Benveniste... Mais la transformation radicale de l'image de la Grèce ancienne à laquelle aboutit son œuvre, Vernant la doit à sa propre expérience du «terrain » et aux difficultés qu'il y a rencontrées. En effet, après la guerre et la résistance, il se met à la rédaction d'une thèse sur le travail chez Platon : c'est l'impasse. Il s'aperçoit qu'il n'y a pas chez ce philosophe de mot pour dire le travail. Si « le terme technè est fondamental, il désigne à la fois le savoir et le savoir-faire mais pas le travail », relate-t-il dans l'Entretien de 1987. Il en tire alors les conséquences :

«Je dois donc me demander si les catégories dont nous nous servons quand nous étudions l'antiquité grecque sont pertinentes. Je m’aperçois que non et je dérive complètement pour aboutir à faire dans les années soixante un tout autre livre que celui auquel je pensais » (Vernant $1987: 25)$.

Le livre en question, c'est Mythe et pensée chez les Grecs (1965, cité : M.P.) dont une partie importante est consacrée au « travail et [à] la pensée technique » (M.P. : 183-247).

Dans la préface de ce livre, J.-P. Vernant explique toutefois le profit que l'on peut tirer de notre " proximité » avec l'homme grec, proximité qui est un fait culturel et le produit d'une histoire. Les œuvres créées par l'homme grec « ne nous sont pas, autant que d'autres, étrangères » et cette familiarité relative nous permet de mieux cerner comment elles sont l'expression d'une activité mentale organisée inséparable « du cadre social et culturel » dont ces hommes « sont à la fois les créateurs et le produit» (M.P. : 9). Or il s'agit de dégager les catégories d'après lesquelles les Grecs pensent leur monde, catégories qui ne sont pas les nôtres, comme leur raison n'est pas la nôtre. Lorsqu'il parle du passage du mythe à la raison, Vernant souligne que « les Grecs n'ont pas inventé la raison mais une raison, liée à un contexte historique, différent de celui de l'homme d'aujourd'hui » (M.P. : 11). Cela est vrai aussi bien pour la mémoire ou l'espace que pour la personne, la représentation ou le travail et la pensée technique. À travers les études qui composent Mythe et Pensée - mais aussi dans les autres travaux de J.-P. Vernant - il s'agit de cerner ce qui nous sépare de l'homme grec pour comprendre « le langage qu'il parle », et même plus, « une architecture d'esprit » que nous ne comprenons pas immédiatement. On voit comment s'exprime une attitude anthropologique même si le mot n'est pas prononcé. Attitude qui est également sensible aux ruptures et aux changements qui s'opèrent à l'intérieur du monde étudié : les comportements se modifient, les catégories ne sont pas données une fois pour toutes, l'architecture de l'esprit n'apparaît pas d'emblée. Il s'agit bien, comme le sous-titre du livre l'indique, d'« études de psychologie historique $»^{2}$.

Le tout premier résultat de ces recherches concerne le thème du travail et la première publication de J.-P. Vernant porte sur « Prométhée et la fonction technique » (Journal de Psychologie 1952 : republié in M.P. : 185-195). C'est un des domaines où la résistance à la discontinuité entre les Grecs et nous est la plus forte : il paraît « évident » que la fonction « unifiée et organisée » du travail telle qu'elle s'accomplit aujourd'hui ne peut être différente de celle qu'accomplissent les Grecs. C'est ici que l'étude anthropologique détruit l'illusion. La technè, l'effort, l'œuvre sont des notions élaborées dans un tout autre contexte. Mythe et religion interviennent pour situer l'œuvre humaine en des lieux insoupçonnables pour nous. Il n'y a pas de grande fonction du travail, chose inconcevable pour le lecteur moderne. Par ailleurs ce que les hellénistes ont appelé - à tort - le blocage de la pensée technique amène à l'absence d'une mécanique appliquée, la nature 
ne pouvant être l'objet de calcul. Les tâches artisanales sont pensées à la fois dans leur rigidité et en tant qu'effort, utilisant tous les ressorts de la ruse pour contourner l'obstacle et réaliser l'objet, fin vers lequel l'effort tend. Tels sont les thèmes saillants, élaborés par J.-P. Vernant, et que nous évoquerons dans cette étude.

\section{La technè et ses œuvres - agriculture et artisanat}

Dans l'article sur Prométhée, Vernant tire déjà toutes les conclusions de sa recherche sur Platon et signale où se pose le véritable problème :

«Dans son ensemble, la fonction [technique] reste mal dessinée et peu systématisée. Rien n'indique [...] l'intérêt pour les motivations du travail - sans doute parce qu'elles sont purement extérieures. Le retentissement de l'action technique et du travail sur l'homme, leur rôle créateur, leur aspect aussi de participation à autrui ne sont pas marqués. La limite entre travail et savoir technique est imprécise. On ne voit pas apparaître l'idée du travail comme grande fonction sociale, comme type d'activité humaine spécifique. On discerne mal ce qui définit le domaine propre du technique ». Ainsi pour les Grecs l'activité technique et le travail « n'ont pas cette forme dense de conduite humaine organisée que nous leur connaissons aujourd'hui » (M.P. : 194-195).

Difficulté lexicale symptomatique : le mot travail n'a pas d'équivalent exact en grec. Ponos s'applique à toutes les activités qui exigent un effort pénible sans que celui-ci soit toujours productif. Quand Héraclès choisit une vie vouée au ponos, la vie vertueuse, il va de soi que son comportement n'a rien à faire avec la vie d'un ouvrier ni avec une quelconque utilité sociale. Ses travaux sont les exploits d'un être d'exception sans commune mesure avec ceux des simples mortels. Ergasia, ergasesthai que nous traduisons par « travail », « travailler », semblent s'appliquer au travail agricole et à l'activité financière l'intérêt (« travail ») du capital ${ }^{3}$. Quant à ergon - l'« œeuvre » - le mot a un sens très large, et concerne l'activité dans son aspect le plus général. « L'ergon, dit J.-P. Vernant, c'est pour chaque chose ou chaque être le produit de la vertu propre», son excellence (M.P. : 198). L'Ergon procède d'ailleurs aussi bien de la production - poièsis - que de l'action - praxis. La production, en effet, concerne le faire de l'artisan et a une fin différente d'elle-même (son résultat) tandis que l'action, selon la définition d'Aristote, a sa fin en elle-même : c'est l'action réussie elle-même qui est sa propre fin.

Comme le montre Vernant, le terme d'artisan, de démiurge, englobe déjà pour Homère tous ceux qui accomplissent une quelconque activité en dehors de la «maison », oikos, en faveur du public, du dèmos : les forgerons, les charpentiers aussi bien que les guérisseurs des maux, les aèdes, voire les mendiants. En prolongeant ces indications, je relèverai pour ma part que le terme technè couvre à l'époque classique un champ immense et s'applique à toute activité finalisée ou considérée comme telle (il comprend également l'activité proprement artistique), même si son résultat n'aboutit pas à la fabrication d'un objet matériel durable : le cithariste comme le gymnaste sont considérés comme des démiurges qui exercent une technè. Le Prométhée d'Eschyle se vante d'avoir apporté parmi les hommes « les arts et les ressources », et cite, à côté de la fabrication de la maison et de l'utilisation des animaux, la divination et 
l'interprétation des songes (Prométhée Enchaîné: 478-500). En fait aucun auteur grec n'a établi un critère suffisamment précis pouvant englober l'ensemble des arts.

J.-P. Vernant commence par aborder la question en situant son investigation autour de l'opposition artisanat/agriculture et en se référant surtout aux Travaux et les Jours d'Hésiode et à l'Économique de Xénophon. D’après son interprétation de ces textes, l'agriculture n’apparaît pas comme un métier. Le paysan d'Hésiode n’a pas le sentiment d'appliquer au sol une technique de culture; son travail, l'ergasia, constitue à la fois une forme de vie morale et d'expérience religieuse. Son « activité laborieuse rigoureusement réglementée » (Vernant 1979 : 60) tend bien sûr à lui faire remplir sa grange mais en même temps à le « rendre proche » de Déméter en obéissant à sa loi. Pour sa part, celle-ci garantit moins la distribution de ses dons qu'un ordre régulier « dans ses rapports avec les hommes » (M.P. : 200). Il s'agit de la sorte pour le paysan de se soumettre à la loi qui commande son commerce avec les dieux. Dans ce cas il n'y a pas lieu de considérer dans la partie des Travaux consacrée à la culture un aspect proprement technique : technique, théologie et morale ne se distinguent pas. Même si l'on considère l'effort humain dans son aspect psychologique, il ne constitue pas une conduite particulière qui a comme but la production des valeurs utiles mais « une forme nouvelle » de l'expérience religieuse. C'est que le travail agricole est la conséquence de la séparation des hommes avec les dieux qui « auparavant » banquetaient ensemble. Cette séparation a pour origine l'ire de Zeus contre Prométhée qui, au moment du partage du sacrifice, a trompé les dieux en leur attribuant les os des bêtes entourés de graisse. À partir de ce moment, les dieux ont caché la nourriture aux hommes et les ont obligés à l'obtenir par un dur labeur.

La position de J.-P. Vernant a été reprise par M. Detienne qui lie la composition du poème hésiodique à la crise agraire et religieuse du viII siècle av. J.-C. Le paysan d'Hésiode vit à une époque où les divisions successorales diminuent les lots familiaux et qui voit les agriculteurs venus d'ailleurs s'installer sur les terres les plus mauvaises. Cette crise agraire s'accompagne d'une crise religieuse et le poème hésiodique appréhende cette dernière en termes de distance qui sépare les hommes des dieux : les attitudes culturelles et ritualistes caractérisant l'agriculture en font une pratique religieuse (Detienne $1963: 325)^{4}$.

Cette position cherche à prendre en compte l'inscription des énoncés concernant le travail du paysan dans l'ensemble des poésies hésiodiques. Sans pouvoir entrer dans les détails de cette analyse, on signalera que l'effort des hommes pour obtenir la nourriture « se rattache à l'esprit de concurrence, de querelle » qui s'est introduit avec Prométhée dans le monde des dieux, la culture céréalière étant étroitement liée au sacrifice (Vernant 1979 : 51 et 61). Il faut signaler en outre l'aspect ritualiste qu'Hésiode fait intervenir tant dans la description des travaux que dans son catalogue des jours fastes pour les exécuter. On voit par conséquent que J.-P. Vernant et M. Detienne contestent l'existence d'un plan «technique » dans l'agriculture hésiodienne, ou du moins récusent son autonomie $^{5}$. En cela ils voient leurs thèses se confirmer par l'Économique de Xénophon, le second grand écrit grec consacré à l'agriculture. Pour cet auteur du Iv siècle av. J.-C., l'agriculture est facile et ne demande ni savoirs spécialisés ni apprentissage : il suffit de regarder les autres qui peinent sur les terres pour l'apprendre. De ce point de vue, on ne peut parler à son propos de technè. L'agriculture n'a pas de secrets et son exercice est une affaire de vigilance, d'epimeleia, « une forme de vertu active faite d'énergie, d'initiative, d'occupation» (M.P. : 202). On ne peut en ce qui la concerne opposer la « connaissance » à l'« ignorance »: «le travail agricole vaut ce que vaut l'homme». Loin de s'assimiler à une quelconque technicité ou à un effort pour la transformation de la nature, l'agriculture 
s'insérant dans un système de représentations religieuses se présente comme « un culte instituant le plus juste des commerces avec les dieux » (M.P. : 204). Xénophon reste ainsi proche d'Hésiode. Pour l'un comme pour l'autre « le travail agricole est pris dans un réseau de croyances religieuses, et tout problème qui le concerne est, pour une large part, théologique » (Detienne 1963 : 56).

Dans cette perspective, agriculture et artisanat s'opposent. Hésiode, déjà, déconseillait aux paysans de fréquenter la forge - aussi bien d'ailleurs que le « parloir de la ville » afin de se consacrer à l'accroissement de la maison (Travaux: 493-495) ${ }^{6}$. Chez Xénophon ce contraste est accentué : L'Économique souligne que pour les artisans la pratique des métiers dépend de recettes gardées secrètes. Leur vie se passe dans des ateliers sombres, ruine le corps et énerve l'âme, les rendant inaptes à défendre la Cité. Par contre, les paysans acquièrent par leur travail un corps plein de vigueur et de beauté : ce sont eux qui vont prendre les armes sans hésitation, quand l'ennemi menace, pour défendre la ville et leurs terres. D'où sa proposition d'exclure des affaires de la Cité ceux qui s'adonnent aux travaux manuels, les banausoi ${ }^{7}$.

J.-P. Vernant souligne avec raison la différence de statut de l'artisan dans le monde de l'épopée et durant la période classique. Dans le monde de l'épopée, la technè s'applique au savoir-faire des démiurges sans qu'on différencie la réussite pratique de l'exploit magique. À l'époque classique la technè repose, par opposition avec le hasard et le don divin, sur l'efficience de recettes positives, sur le savoir acquis par l'apprentissage, se constituant en règles du métier. Les techniques sont laïcisées. L'activité artisanale répond à une exigence « économique »-être toujours au service d'autrui, produire ce que les autres peuvent utiliser et consommer. Elle est, il faut bien le souligner, essentiellement citadine :

«C'est en fonction du fait urbain de la division du travail que se définit [...] une notion positive de la technè : activité spécialisée [et] ensemble de règles permettant de réussir dans le domaine de l'action » (M.P. : 207).

Tous les textes du $v^{e}$ et Iv siècle av. J.-C. s' accordent sur le caractère urbain des arts et sur la nécessaire division des tâches - division que l'on ne peut confondre avec ce que l'on appelle depuis la révolution industrielle division manufacturière du travail. Il s'agit pour la Grèce de la division entre métiers : seul le cordonnier - celui qui se limite à sa tâche et à elle seule - peut fabriquer de bonnes chaussures, le charpentier fabriquer les lits parfaits et le forgeron les outils adéquats aux opérations envisagées. Ce faisant, l'artisan s'isole, s'enferme dans sa seule capacité, sa compétence propre : celui qui sait faire une chose et ne sait faire que celle-là.

Cette pensée préoccupée de l'usage, de l'utilisation du produit, du service que l'artisan procure, ignore la productivité moderne - en l'absence d'ailleurs d'un marché généralisé. C'est cette division des tâches qui apparaît comme étant à l'origine de la Cité. La Cité doit sa naissance, comme l'explique Platon (République : 369b), « à l'impuissance où chacun de nous se trouve de se suffire à lui-même » et au besoin que chacun éprouve d'un grand nombre de gens : chacun d'entre eux exerce un travail particulier et satisfait les besoins de tous les autres par le produit de celui-ci. Ainsi, la pluralité des métiers et des capacités rend possible la réunion des hommes dans un lieu - la synkatoikèsis - à l'intérieur duquel la satisfaction mutuelle des besoins peut se réaliser. Ceci ne suffit pas à faire fonctionner la Cité qui ne se réduit pas à une somme d'artisans - et de cultivateurs, si l'on suit le texte platonicien. C'est une condition nécessaire mais non suffisante - et cela est vrai aussi bien pour Platon et Aristote, critiques envers le régime démocratique, 
que pour Protagoras et certains sophistes, défenseurs des pratiques de l'assemblée. Il faut un liant pour que la Cité puisse exister et vivre, et cela relève d'une capacité particulière, celle concernant les affaires politiques. Savoir si tous les citoyens la possèdent ou non constitue l'enjeu des affrontements entre les différents courants politico-théoriques ${ }^{8}$.

Chaque art présuppose ainsi pour celui qui l'exerce une capacité, une nature particulière et pour celui qui utilise son résultat, un besoin. À la pluralité des métiers correspond une pluralité des natures. Dès lors, le « spécialiste » doit rester enfermé dans les limites de ce qu'il sait et peut réaliser. Il n'y a pas, pour cette pensée, un ensemble de métiers qui s'articuleraient entre eux pour former un système fonctionnant à partir de sa propre légalité. L'activité artisanale en elle-même n'est pas le libre déploiement des facultés humaines. En effet chaque technè vise à produire dans la matière une « forme », un eidos (la maison, le navire...) par la mise en œuvre d'une capacité utilisée quand il convient et comme il convient. Cette pensée adopte une interprétation totalement opposée à celle que nous, modernes, attribuons à l'activité technique. Non seulement l'artisan est censé viser une "forme » préexistante à réaliser, mais le centre de gravité de cette activité est déplacé. On considère moins le processus de fabrication (poièsis) que l'usage qui est fait de son produit. Ainsi s'impose une forme ou un modèle qui n'est pas une invention du fabricant : celui-ci doit se conformer au modèle soit en fixant les yeux sur lui, soit en faisant confiance à l'usager qui « est mieux placé » pour connaître la forme parce qu'il est le seul à avoir l'usage du produit. C'est par conséquent le modèle ou l'usager qui occupent la place principale par rapport à la fabrication elle-même. La finalité technicienne ne se différencie pas en ce sens de la finalité propre à la biologie et à la physique.

Rappelons que l'objet fabriqué n'est pas totalement assimilable à l'objet naturel. Dans le cas d'une homologie complète entre les deux, l'objet fabriqué serait l'effet d'une spontanéité naturelle, sinon du hasard. «La production humaine », explique Vernant, « obéit à une finalité intelligente, alors que les processus naturels s'accomplissent au hasard et sans prévision » (M.P. : 213). Il ajoute, à juste titre, que la pensée grecque ne considère comme naturel ni les procédures et les règles de l'art ni leurs produits. L'opération artisanale par contre s'inscrit dans le cadre de la nature : elle n'intervient pas pour la modifier et instaurer un autre ordre - un ordre humain. L'artisan ne transforme pas la nature, il « exécute » ce que la nature est « impuissante à produire » - apergasesthai - selon la proposition d'Aristote. Aristote souligne, en effet, que si « les choses naturelles » - ta physei - n'étaient pas produites par l'art mais également par la nature, « elles seraient produites par l'art de la même manière qu'elles le sont par la nature ». Si les procédures et les règles techniques ne sont pas appréhendées comme naturelles, c'est que la production imite la nature (Physique II, 1981b : 13-14 et 16-17). Cette mimèsis donne lieu à une délibération, à une évaluation des moyens intervenant dans la fabrication pour « avoir le dessus » vis-à-vis des éléments auxquels l'effort artisanal s'applique ${ }^{9}$.

Cela dit, l'opération artisanale ne se différencie pas, pour le reste, du processus naturel. Ainsi le traité hippocratique Du régime veut montrer dans une longue digression (Littré VI : 486-496) que les technai utilisées par les hommes « sont semblables à la nature humaine ", voire à la nature tout court. De l'art, écrit qui veut défendre la médecine en tant que technè, essaie de montrer que l'art existe à la manière des « réalités naturelles » (Littré VI : 4 et 7-8), la connaissance s'acquérant « au fur et à mesure que les arts sont montrés ». Que l'art médical existe lui-même, cela se prouve avec les malades qui se guérissent sans demander l'aide du médecin : ce faisant, ils suivent, sans le savoir, les procédures inscrites dans la « réalité » de l'art médical (Littré VI : 8). 
Finalement la formalisation par la philosophie de l'ensemble de ces représentations appliquera à la technique les mêmes catégories qu'à la nature. Aristote, qui élabore la forme la plus achevée de cette conception, pense les productions techniques au moyen de la quadruple causalité : l'artisan, cause motrice, opère sur un matériau, cause matérielle, pour lui donner une forme, cause formelle qui est en même temps la fin de l'opération, ce en vue de quoi l'opération a eu lieu - la cause finale ${ }^{10}$. L'artisan, d'après cet ordre de représentation, ne transforme pas la nature, ne se trouve pas à la source d'une invention (nouveauté radicale par rapport aux données existantes) qui le « livrerait désarmé au hasard » (M.P. : 242). Il se situe à un point bien précis de la géographie des essences fixes, l'ensemble des arts dont chacun demande une compétence particulière et exclusive, et a recours aux recettes et aux procédés transmis par la tradition. De ce point de vue il n'est pas faux de dire que « la technè artisanale n'est pas un véritable savoir » (M.P. : 242) : l'artisan ne possède pas un schéma explicatif de son faire. Toutefois, dans son activité, l'artisan affronte un devenir et son art consiste à saisir la circonstance favorable pour l'accomplissement de son ouvre : il est, en quelque sorte, l'esclave du kairos, de ce moment fugitif pendant lequel il peut réaliser le produit. Aux prises avec l'instabilité du devenir, l'artisan se trouve dans l'obligation de recourir à un expédient pour résoudre cette difficulté. Fixité, routine d'une part, ruse de l'autre : c'est en cela que consiste l'ambivalence de l'activité artisanale. Au moment où il écrit ses « Remarques sur les limites de la pensée technique » (Revue d'histoire des Sciences 1957, republié in M.P. : 228-247), J.-P. Vernant pense qu'à « l'âge de la cité » l'astuce et l'ingéniosité paraissent avoir quitté les ateliers et les échoppes ; la ruse, la mètis, inspire plutôt le sophiste ou l'« ingénieur ». Cette opposition entre les arts ne va pas sans poser problème, et il faudra attendre les études consacrées aux Ruses de l'intelligence pour amorcer une solution.

\section{Les mécaniciens - ingénieurs}

Restons-en pour le moment au problème des ingénieurs et des mécaniciens. Les Grecs, selon les analyses de J.-P. Vernant - confirmées par les travaux de M. I. Finley $(1984,1975)$ - ne sont pas novateurs. Leur outillage et leurs savoirs sont les mêmes que ceux de l'Orient et les améliorations importantes apportées ne dépassent pas le cadre du système technique fixé, pour l'essentiel, à l'époque classique. Il s'agit de l'application de la force humaine et animale à travers les instruments. En brossant rapidement ces traits, J.-P. Vernant ne cherche pas à démontrer que tout reste immobile pendant la période hellénique. Les travaux consacrés à la guerre qu'il a dirigés (1986b) montrent au contraire des changements : il y a passage d'une bataille où les nobles chevaliers jouent le rôle prépondérant, à la phalange hoplitique dont la tactique présuppose la solidarité entre « égaux » - les citoyens en armes - et à une moindre intervention de la cavalerie. Par ailleurs des modifications concernant « la masse compacte de la phalange », l'armement et le recrutement des soldats interviennent pendant l'époque hellénistique. Encore faut-il s'interroger sur la signification de la guerre et l'« évolution » de cette signification. On voit apparaître de nouveaux modes de fortifications, on a recours aux « machines » pour détruire les murs de la cité, on fait de plus en plus appel aux mercenaires. On voit aussi apparaître « une spécialisation croissante de la chose militaire », la guerre devenant une activité à part, une 
technè ayant « en elle-même sa finalité et ses moyens », cessant par là d'être le fait de la Cité, avant même que celle-ci ne disparaisse (Vernant 1968b : 20 et passim). On connaît, pour passer à un autre domaine, l'intérêt de certains présocratiques pour la technique et ses réalisations : on connaît également les modifications intervenues dans l'architecture ou dans les mines, etc. ce qui a amené des savants, comme le regretté B. Gille, à soutenir l'existence de «techniques en progrès » $(1980: 28,204-15)$. Toutefois, comme ce «progrès » est loin de couvrir tout l'espace du domaine technique, on voit surgir parmi les hellénistes au cours des années cinquante l'idée d'un blocage de la pensée technique chez les Grecs. Dans l'article de 1957, Vernant conteste l'existence d'un tel blocage qui serait dû soit au désintérêt ou au mépris des Grecs pour le fait technique soit à l'existence d'une main-d'œuvre d'esclaves bon marché empêchant l'avènement du machinisme.

Comme on le sait, la question se pose à propos de certaines réalisations des $v^{e}$ et $\mathrm{IV}^{e}$ siècles av. J.-C. qui ont trouvé leur prolongement dans les efforts des « mécaniciens » alexandrins. Il y a eu quelques inventions importantes - pompe à incendie, orgue hydraulique, engins de guerre, horloge hydraulique, presse à vis - et la construction de quelques « automates » considérés comme des objets merveilleux. Les éléments de ces dispositifs sont les cinq machines simples ainsi que la valve, le cylindre, le piston, la roue dentée... On utilise la torsion des câbles, l'élasticité des métaux ou la compression de l'air et des liquides. Ces dispositifs servent surtout à démultiplier la force humaine ou à mettre en place des instruments de mesure relativement complexes mais peut-être mal adaptés à leur fonction. L'horloge de Ctésibios qui utilise l'écoulement d'un débit d'eau régulier demande une constante intervention humaine en raison de la différence de durée des heures, telle que la concevait l'antiquité. De toute manière, l'idée que ce type de mécanismes puisse commander aux forces de la nature n'apparaît pas. La question qui préoccupe J.-P. Vernant concerne la capacité ou l'incapacité à appliquer une procédure scientifique à cette mécanique ${ }^{11}$. En effet, la science pour les Grecs porte sur les essences immuables, tandis que sur le terrain de l'expérience le savoir ne peut être obtenu que par tâtonnements. Les Grecs s'occupent peu de l'ajustement des modèles à la réalité technique, les résistances et les frottements n'étant pas calculables (et cela, il est vrai, pour longtemps). Dans le cas de Baroulkos, un traité d'Héron d'Alexandrie consacré à un système d'engrenages destiné à soulever un objet pesant par une force nettement 
moindre, la solution proposée est irréalisable même si le schéma théorique est valable, l'articulation du théorique et de la pratique faisant défaut. Par contre, sur le «plan unilatéral » de la théorie - comme c'est le cas des Questions mécaniques attribuées à Aristote -, on utilise une démarche analogue à celle de la sophistique : comment dominer une force supérieure par des moyens faibles. Comme l'orateur maîtrise les procédés qui permettent aux arguments les plus faibles de l'emporter, ou en tout cas d'équilibrer les arguments les plus forts, la mécanique est conçue comme le domaine où « le plus petit domine le plus grand» (Pseudo-Aristote, Questions mécaniques : 847a, 22). En ramenant toutes les procédures mécaniques à la combinaison des cinq machines simples, on veut comprendre la manière selon laquelle « on peut calculer des forces inégales données par avance, dont l'une est la force humaine, l'autre un poids aussi grand soit-il» (M.P. : 240). La proposition de J.-P. Vernant concerne Héron et montre comment celui-ci ne peut se détacher de la perspective théorique pseudo-aristotélicienne. Le modèle trouve vite ses limitations et son achèvement sans arriver à produire une mécanique appliquée.

Dans le domaine de la balistique et de la pneumatique, la puissance de la physis n'est pas calculable. Même si l'on arrive à produire des tables et des modules - lesquels ont attiré l'attention de B. Gille -, il faut encore prendre en considération comment tables et modules se sont établis : par les tâtonnements et par les ajustements successifs provenant de la simple empirie. De surcroît, on peut se demander quelle était la diffusion effective de ces dispositifs alexandrins : on est en droit de soupçonner, par exemple, que les horloges de Ctésibios n'étaient construites qu'à de rares exemplaires.

Mais Vernant essaie de dégager la logique qui domine les expériences et les limites de la pensée technique hellénistique. Le blocage de cette pensée ne s'explique pas par des raisons d'ordre social ou par la prédominance de l'attitude contemplative des philosophes et des savants. Vernant oriente son investigation vers la manière dont les éléments d'un système technique s'articulent d'une façon cohérente et solidaire. Ainsi, il veut expliquer les limites de la pensée qui se préoccupe de la technique en la renvoyant à la globalité du système technique grec :

«Chaque système technique a sa pensée propre. L'utilisation d'un outil, la mise en jeu d'une technique, sont des faits intellectuels solidaires d'une structure mentale en même temps que d'un contexte social : non seulement ils dépendent de la forme et du niveau général des connaissances mais ils impliquent tout un ordre de représentation ». (M.P. : 233).

C'est un véritable tournant qui est ainsi opéré dans la compréhension de la technique grecque. Il s'agit de montrer les connexions entre les représentations concernant le travail, les modalités des opérations et les limites à l'intérieur desquelles cette technique fonctionne.

\section{L'intervention de la mètis}

J.-P. Vernant et M. Detienne ont étudié, à côté de la conduite proprement technicienne, une attitude qui concerne l'ensemble de l'action : celle de la mètis (la ruse) qui recouvre en partie les activités artisanales. En fait le domaine de la mètis est immense - « l'univers culturel dans toute son étendue »- et le rôle de l'artisan est loin d'être abordé dans les 
Ruses de l'intelligence (1974, cité : R.I.) sous tous ses aspects. L'homme à mètis, aux prises avec la réalité changeante du devenir, recourt à l'expédient, à la tromperie, au piège, aux moyens les plus obliques et les plus ondoyants pour atteindre son but. L'intelligence rusée, pour arriver à ses fins, prend un chemin détourné.

C'est une démarche de type agonistique dans laquelle tous les moyens sont bons. De toute manière la mètis est :

« [...] une catégorie mentale, non une notion [...]. Car les formes d'intelligence rusée, d'astuce adaptée et efficace que les Grecs ont mises en œuvre dans de larges secteurs de leur vie sociale et spirituelle [...] ne font jamais l'objet d'une formulation explicite, d'une analyse en termes de concept, un exposé d'ordre théorique » (R.I. : 9).

On trouve déjà dans l'lliade la situation type (Il. 23 : 206 et suiv.) : l' aurige Antiloque, possédant des chevaux médiocres, est battu d'avance dans une course où les concurrents sont redoutables. Il a recours à un tour sans loyauté ; profitant du brusque resserrement de la piste, il pousse son char en oblique devant celui de Ménélas qui, surpris, doit retenir ses chevaux et laisser son adversaire prendre de l'avance. Devant l'aporie qui caractérise les données initiales, la mètis est la puissance qui ouvre le chemin vers une solution avantageuse. Le modèle fonctionne partout et cela pendant dix siècles. On le retrouve dans les écrits concernant les combats entre dieux pour acquérir la souveraineté, la pêche, la chasse, le pilotage des navires, la médecine, ... La mètis « ne se manifeste pas [toujours] pour ce qu'elle est », d'où sa méconnaissance par les savants modernes qui, en ne reconnaissant pas son rôle, suivent en cela les philosophes anciens. Méconnaissance qui est un refus de reconnaître que la mètis, à travers ses multiples manifestations constitue bel et bien « une forme d'intelligence et de pensée, un mode de connaître » (R.I. : 9) ${ }^{12}$.

Il va de soi que nous n'allons pas suivre tous les linéaments de J.-P. Vernant et de M. Detienne; nous nous limiterons à quelques cas exemplaires. Toutefois il faut préciser d'emblée que la mètis n'est pas présente partout : il y a des dieux à mètis et d'autres sans, comme il y a des conduites utilisant tous ses moyens et d'autres pas. L'intelligence rusée déploie ses pouvoirs sur « un terrain mouvant dans une situation incertaine et ambiguë » où l'homme à mètis épouse le caractère des réalités fluides et mouvantes en devenant lui-même fluide et polymorphe. Il dépasse ainsi le présent en prévoyant l'avenir (R.I. : 21 et 32). Cette forme d'intelligence cherche à s'orienter dans le changement et l'instable et essaie par tous les moyens de maîtriser le devenir (R.I. : 56) afin d'en tirer profit. L'ambition exprimée par les mythes, les histoires légendaires et les procédures techniques est non de transformer le donné mais bien de le juguler. Devant l'imprévisibilité du cours des événements, il faut piéger, enfermer, immobiliser ce qui peut échapper ou disposer de forces supérieures, le tenir jusqu'à ce qu'il s'avoue vaincu. De surcroît, l'intelligence rusée se trouve dans une situation où l'on ne connaît pas la totalité des événements et des forces qui peuvent surgir. L'opération est engagée à travers les ambivalences qui caractérisent cette situation; elle doit se modifier, changer de parcours en employant tous les trucs dont dispose celui qui les effectue. La mètis affronte souvent une autre mètis. Elle doit donc « renverser à son avantage les puissances rusées qui méditent de retourner contre elle, ses pièges et ses filets » (R.I. : 291).

La conduite-modèle de l'homme rusé se retrouve ainsi dans le cas les plus inattendus, les plus variés. C'est bien sûr la chasse, la pêche mais aussi le pilotage des navires, la rhétorique et l'art de gouverner les cités. L’Tliade (23, 315 et suiv.) le montre déjà : «C'est par la mètis, plus que par la force, que vaut le bûcheron. C'est par la mètis, que sur la 


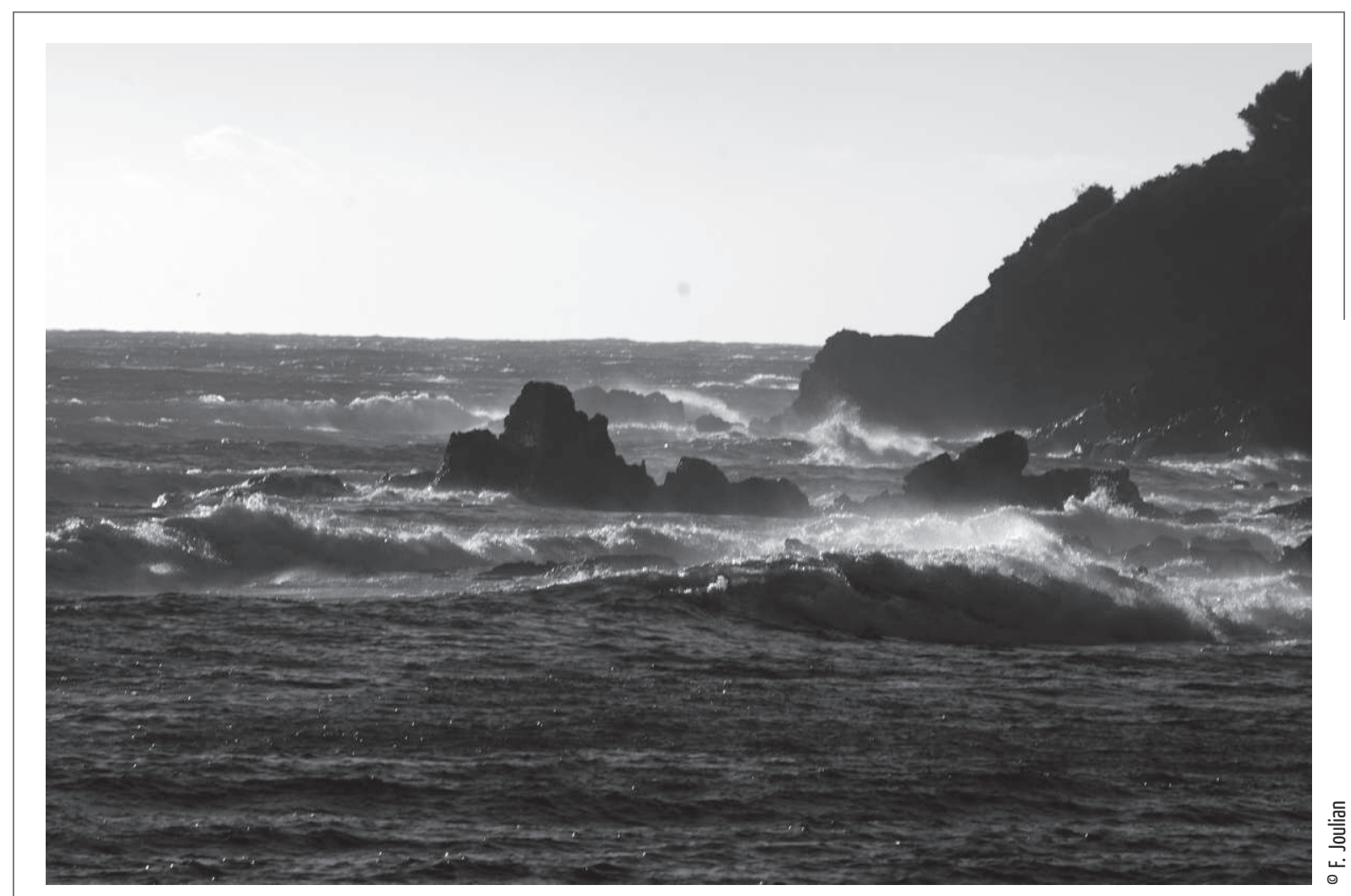

\section{Accostage difficile}

Cliché, 2007.

mer vineuse l'homme guide le bâtiment de course [...] c'est par la mètis que le cocher l'emporte sur son concurrent ». Sans doute, pour la pensée mythique, c'est Athéna, divinité à mètis, qui préside à l'œuvre de la construction. C'est aussi que la mètis présuppose la justesse du coup d'œil. En pleine époque classique, Aristophane dit à propos de l'artisan qui fabrique la lampe qu'il doit, parmi d'autres qualités, avoir « l'œil sûr » (Assemblée des femmes : 1 -2). À la vérité toutes les technai ont plus ou moins recours aux comportements à mètis. Si la mètis fait le bon bûcheron, la même mètis intervient dans la pratique du médecin. La maladie, "puissance douée de métamorphose » ayant son rythme propre, est traversée par « les jours critiques », d'après toute la tradition hippocratique. Ces jours critiques constituent « le point fugitif où la technè du médecin, ce chétif, peut triompher des puissances hostiles » de la maladie (R.I. : 298). Assez paradoxalement - mais conformément à la logique de la mètis -, le savoir médical, à partir au moins du ve siècle, montre des affinités avec la navigation : le rapprochement s'effectue en mettant en parallèle le pilote qui tient le gouvernail et le médecin qui veut sauver le malade du danger que la maladie présente. « La plupart des médecins ressemblent aux mauvais pilotes » dit l'auteur de l'Ancienne médecine, qui a des comptes à régler avec ses confrères ; « tant que le calme règne, leurs fausses manœuvres ne sont pas apparentes ; mais viennent un mauvais orage et un vent impétueux, ils laissent périr le bâtiment [...]. 
Il en est de même des mauvais médecins, qui forment le plus grand nombre » (éd. Littré, 590). Ainsi les parallélismes se multiplient et se développent également entre rhétorique, politique, médecine et navigation ${ }^{13}$.

Sur le plan du mythe un des exemples centraux choisi est précisément la navigation: c'est à partir de l'analyse de ces récits que se tisse tout un champ du vocabulaire de la mètis et que s'organise la configuration de cette forme d'intelligence. M. Detienne et J.-P. Vernant arrivent de la sorte à saisir un univers mental, un ensemble de représentations qui jouent également dans le domaine de l'activité technique. Il ne s'agit pas bien sûr d'utiliser le matériel mythique pour arriver à saisir la « réalité » de cette activité. Toutefois le mythe contribue à sa manière à organiser la représentation du technique ; bricolé à partir d'éléments disparates, il puise ses matériaux, en partie tout au moins, dans la pratique technicienne. Athéna la polymètis, dont le domaine d'intervention est la guerre comme le monde artisanal (R.I. : 171), préside et donne des conseils aux Argonautes pour la construction de leur navire et les aide à parcourir la mer pleine de dangers. Comme on le sait le navire argonautique doit traverser les roches errantes. L'oiseau envoyé par Athéna (identifié à la corneille de la mer) s'élance à travers ces roches. Ce qui constitue l'indice que les navigateurs peuvent traverser le passage. Le rôle de l'oiseau, disent nos auteurs, «ne devient parfaitement intelligible que par référence à certaines pratiques

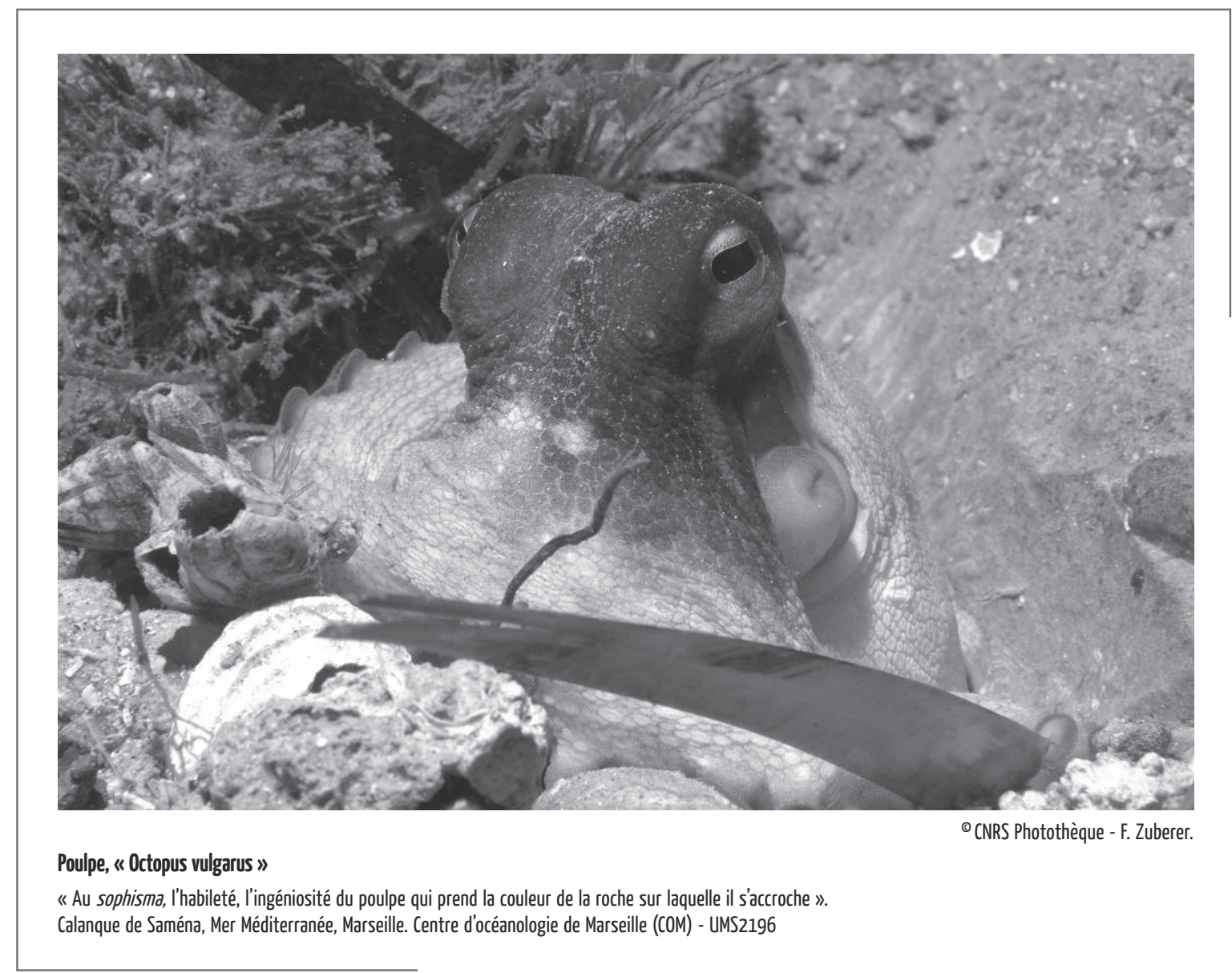


de la navigation dans l'antiquité ». En effet, les navigateurs emportaient avec eux des oiseaux qu'ils lâchaient quand ils voulaient connaître la direction de la terre (R.I. : 208). La séquence mythique contribue ainsi à élucider les catégories à partir desquelles l'action technique devient intelligible pour les Grecs.

Mais les interventions des divinités - comme leur combat pour le pouvoir - aboutissent à un terme qui représente une stabilisation du « cours naturel » : les rochers ne bougent plus, Zeus établit sa prédominance. Pour les humains, par contre, l'affrontement contre ce qui ne reste jamais semblable à soi continue.

Chasser, pêcher, soigner la maladie, s'opposer à l'ennemi ou affronter ses concitoyens dans l'agora, c'est utiliser ruses et expédients, se conformer au même patron agonistique. Le pêcheur doit déjouer les mille « inventions » des poissons : « souvent » ceux-ci « s'échappent, lorsque l'hameçon déjà les tenait [...] ; vainqueurs dans le combat de la ruse, souvent ils triomphent des artifices de l'homme » disent les Halieutiques d'Oppien (citées in R.I. : 39), un auteur du II siècle ap. J.-C. ${ }^{14}$. Au sophisma, l'habileté, l'ingéniosité du poulpe qui prend la couleur de la roche sur laquelle il s'accroche ou de la seiche qui se cache dans la nuée (selon la terminologie grecque) qu'elle laisse échapper, répond l'inventivité du pêcheur. Les mots utilisés ne laissent pas de doute : sophisme, dolas, tours, technè se retrouvent aussi bien du côté des pêcheurs que des êtres marins : la ruse de l'homme n'est ainsi qu'une réplique à la ruse déployée par l'animal. De même, face au chasseur, le renard qui se creuse une demeure à « sept portes différentes », habile à déjouer pièges et traquenards, fait le mort. Pour le piéger « il rie faut pas compter [...] ni sur les lacets, ni sur les rets, car il n’a pas son pareil pour flairer l'embûche » (Cynégétiques IV, 449-460). C'est la mètis qui va venir au bout de la proie : Oppien annonce qu'il va énumérer les stratagèmes, les pièges, les artifices du dolas que les chasseurs utilisent. Les auteurs grecs soulignent aussi que l'effort de l'homme pour capturer les animaux se superpose à l'effort incessant des animaux eux-mêmes pour capturer leurs congénères en utilisant artifices, tromperies et même la technè qui leur est propre. Le renard se retourne brusquement sur sa proie quand elle s'approche de lui ; son homologue marin, « le poisson-renard », apparaît comme son double dans sa conduite du retournement : « il déplie ses organes intérieurs, il les retourne » (Elien, De la nature des animaux, IX : 12) ; « il retourne (son corps) en sorte que l'intérieur devient extérieur » (Plutarque, L'intelligence des animaux, 977b). Inversement, les chasseurs, selon Plutarque, doivent rester silencieux et invisibles pour se faire eux-mêmes des pièges. Dans cette lutte sans merci " pour triompher des êtres pleins de ressources ", " chasseurs et pêcheurs doivent être d'une mètis plus grande, avoir dans leur sac plus de tours que ne peuvent en aligner leurs victimes » (R.I. : 39). Contre la «nuée » de la seiche, pour ne prendre qu'un exemple, ne peut triompher que la «nuée » des filets.

Les écrits cités décrivent toute une série de comportements fantastiques des animaux. Ils ne sont pas, d'ailleurs, les premiers à les mentionner ; Aristote, déjà, en informait son public. Ces comportements, les auteurs anciens ne les ont pas trouvés dans la nature « mais dans leur propre pensée, dans la conception qu'ils se faisaient de la mètis, de ses moyens, de ses effets ». Plus encore : à travers la représentation des animaux rusés, on voit précisément se circonscrire une forme d'intelligence humaine. Ils ne présentent pas seulement la même duplicité que chez les hommes essayant de les attraper ; leur comportement sert de modèle à «l'intelligence en tentacules », à l'esprit d'embûche du sophiste et du politique. Les analyses de J.-P. Vernant et de M. Detienne montrent comment les astuces des animaux, telles qu'elles sont décrites dans toute une série de textes, servent de modèle à toute une série de personnages. Ulysse le polymètis 
est surnommé « un poulpe », les discours ondoyants des sophistes enlacent leurs adversaires comme les tentacules du poulpe et s'adaptent aux circonstances de la vie politique « en changeant de visage ». La guerre n'est pas loin avec ses stratagèmes et ses tromperies : un texte comme la Poliorcétique d'Elien le tacticien y consacre une grande part. La guerre se présente dans le discours grec comme une activité apparentée à la chasse et à la pêche (on enferme les navires ennemis dans une nasse) ${ }^{15}$. Mais au-delà, c'est un rapport étroit qui s'établit avec « les techniques les plus anciennes» celles qui ont recours au tissage mais aussi aux ajustements (sous la rubrique «tresser », Platon englobe tissage et charpenterie : Lois III : 679a; Politique : 283b).

Cette articulation entre des pratiques fort éloignées les unes des autres constitue l'indice qu'on est en présence d'une intelligence qui est «impliquée directement dans les difficultés de la pratique avec tous ces aléas, confrontée à un univers de forces hostiles, déroutantes parce que toujours mouvantes et ambiguës » (R.I. : 52). C'est ce qu'Aristote

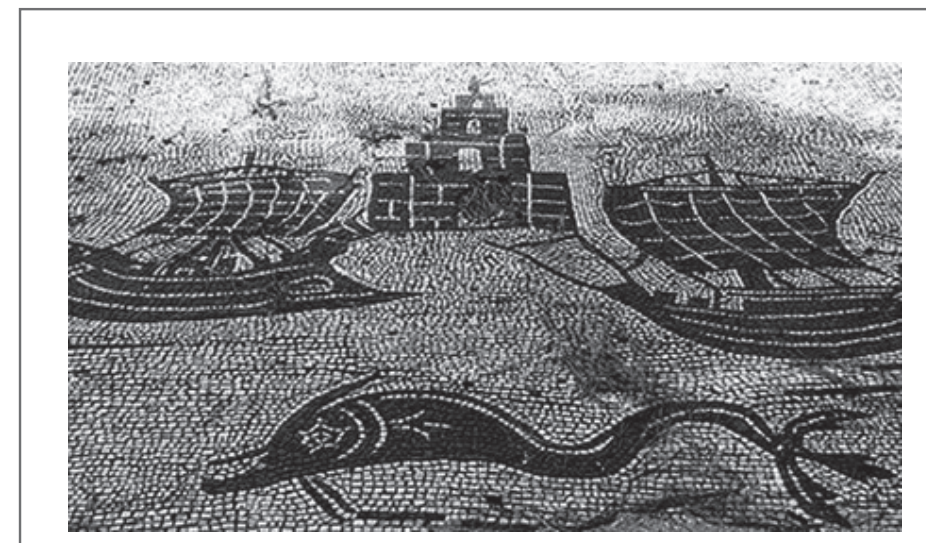

Ostia Antica souligne quand il dit que dans la navigation, il n'y a pas de savoir général de tous les cas particuliers, pas de connaissance de tous les vents qui sillonnent la mer (Éthique à Eudème VIII, 1274a : 5-7). En généralisant cette remarque, il caractérise les choses qui tout en se produisant avec fréquence (epi to polu) « demeurent incertaines dans leur aboutissement » (Éthique à Nicomaque III -1112b : 8). Pour les aborder, Platon fait appel à la « visée juste », l'eustochia, mot qui se rapporte à l'habileté de l'archer. Viser juste et agir rapidement, c'est s'adapter à un monde caractérisé par le mouvement incessant. Mais si Platon s'intéresse à toutes ces composantes de la mètis, c'est pour condamner tout savoir conjectural - le savoir de ce monde-ci - au profit d'une vérité immuable.

Le dossier ouvert par les Ruses de l'intelligence est loin d'être clos. En ce qui concerne le point de vue qui nous intéresse dans ces pages, beaucoup reste à faire. Les articulations ou les rapprochements indiqués par J.-P. Vernant et M. Detienne entre toute une série d'activités (navigation, pêche, tissage... ) restent à préciser et doivent s'étendre à des domaines voisins. C'est la tâche des hellénistes que d'enrichir ce dossier ; surtout sur un point : comment fonctionne la mètis à l'intérieur du «savoir d'un habile artisan, au sens traditionnel [...] un savoir organisé, avec ses règles et ses procédés transmis d'une génération à une autre dans des corps des métiers comme les forgerons et les charpentiers » (R.I. : 302). Forgerons et charpentiers qui ont recours, comme les Ruses de l'intelligence l'indiquent, aux moyens de la mètis. Le modèle de celle-ci comportant le contournement d'un obstacle, tous les arts s'y trouvent d'une manière ou d'une autre impliqués. La préface du livre le signale expressément quand elle fait étendre le domaine de la mètis à l'intelligence pratique «affrontée à des obstacles qu'il faut dominer en rusant pour obtenir le succès dans les domaines les plus divers de l'action » (R.I. : 8). Il est souligné que ce type d'intelligence se maintient « dans de vastes secteurs : la politique, l'art militaire, la médecine, les savoir-faire artisanaux », que la philosophie du Iv siècle tend à déprécier et à dévaloriser par rapport à ce qui constitue désormais «le foyer de la science hellénique » (R.I. : 9). 
Cette dévalorisation, conséquence d'une démarche qui vise les essences fixes et le monde sans devenir du supra-lunaire, concerne l'incertitude relative aux résultats des opérations concrètes. Or les opérations concrètes, même les plus humbles, comportent un minimum de mètis pour qu'elles puissent se réaliser. Telle est, à mes yeux tout au moins, la conséquence des investigations de J.-P. Vernant et de M. Detienne. On doit toutefois signaler que les énoncés se rapportant à la mètis sont à double tranchant. En insistant sur l'imprévisible, le polymorphe, ils font appel à une intelligence toujours aux aguets qui invente toutes sortes de procédés ingénieux pour pouvoir les dominer. Ces énoncés offrent en même temps un catalogue de comportements et, disons-le, de recettes visant à dominer l'adversité. On dirait même que la fonction de ces énoncés consiste à solidifier les actions multiformes, les comportements auxquels la mètis préside. Ainsi les textes comme les Halieutiques et les Cynégétiques d'Oppien, en énumérant tous les « cas prévisibles »- les ruses des animaux qui se répètent sans cesse et les attitudes à adopter à leur égard - enferment le pêcheur et le chasseur dans une certaine rigidité. Il se dégage de ces écrits une tendance à l'exhaustivité, au recensement de tous les comportements imaginables envers l'« objet », l'« obstacle » à vaincre - qu'il soit animal, humain ou matériel. Ce faisant, ces écrits introduisent ce qui rend possible l'activité de la technè, une « réglementation » à laquelle une action efficiente doit se conformer. Entre la mètis polymorphe et la routine, la distance se rétrécit et tend à s'annuler : en présentant tous les technasmata imaginables, la ruse à laquelle on a recours devient alors un simple procédé connu d'avance et se range parmi les savoirs et les savoir-faire que l'homme exerçant un art - humble ou noble - aura à sa disposition.

Au terme de ce parcours, on voit toute la complexité que recouvrent les catégories à partir desquelles les Grecs abordent travail et technique. Pas de terme générique pour le premier, une extension immense pour le second. La pensée grecque aborde la technè sous deux angles opposés. D'une part l'extrême rigidité de l'artisan réalisant son œuvre d'après les modèles imposés et selon des procédures routinières sans aucune inventivité, d'autre part son extrême souplesse, son adaptation au moment, à l'occasion fugitive et le recours aux tours multiples de la mètis. Peut-être pourrait-on reprendre les conclusions de l'article de Vernant de 1957 : dans le premier cas il est question des artisans travaillant dans les ateliers, les banausoi, ceux qui exercent « les arts de peu de valeur ", selon les termes de Xénophon; dans le second il s'agit des sophistes, des mécaniciens, des pêcheurs ou des bûcherons. Mais à toutes ces activités les Grecs accordaient le nom de «techniques». Il devient de la sorte difficile d'introduire des coupures rigides entre des pratiques souvent voisines, ou considérées comme telles par cette pensée. Les métiers dont J.-P. Vernant parle - en fait l'ensemble des métiers exercés dans la société grecque - se situent à l'intérieur d'une hiérarchie : le fabricant de chaises ne se trouve pas sur le même plan que le médecin ou le rhéteur. Mais pour expliciter la notion de technè, les auteurs anciens font appel aux uns et aux autres. Cela fait apparaître que la hiérarchie en question, bien qu’admise, n’a jamais été réellement 
abordée alors qu'elle soutient les énoncés concernant la technè. Il en va de même de la rigidité et de la ruse en œuvre dans les pratiques techniciennes. À la vérité, si l'on veut bien tirer toutes les conclusions de la démarche de J.-P. Vernant, la technè apparaît pour ce qu'elle est, c'est-à-dire ambiguë. De cette ambiguité concernant la technique, la pensée grecque n’a jamais pu se départir.

\section{NOTES}

* Remerciements. Dans cet exposé nous essayons de suivre de plus près la démarche de J-P. Vernant en citant aussi bien ses textes que ceux auxquels il se réfère. Toutefois, il ne s'agit pas d'un compte rendu. Nous voulons montrer la cohérence d'une approche que nous considérons comme valable dans ses grandes lignes. C'est en cela que consiste notre principal objectif. Nous sommes ainsi obligés de ne pas tenir compte des prolongements du travail de Vernant ni des « reprises critiques » auxquelles ses travaux ont donné lieu. (Je remercie Jean-Michel Rey pour sa lecture et ses commentaires de ce texte).

1. Voir également sa préface à un autre recueil d'articles du même auteur (1983: 7-13) et la contribution de R. Di Donato. L'étude de S.C. Humphreys (1978) insiste surtout sur l'enracinement durkheimien de la pensée de L. Gernet. Pour I. Meyerson on consultera sa thèse (1948) et l'article de P. Malrieu (1987).

2. Hommage évident à I. Meyerson qui a forgé la notion, notion qui est loin d'être, à nos yeux, convaincante. Toutefois l'influence de Lévi-Strauss est déjà présente dans certains textes de Mythe et Pensée.

3. R. Descat $(1986: 17)$ conteste cette interprétation qui lui paraît « arbitraire ». Il se peut que J.-P. Vernant force le trait pour souligner la diversité des domaines auxquels s'applique le mot tout en étant absent là où l'on pourrait s'attendre à son emploi. Pour ponos il faut consulter N. Loraux (1989) qui traduit ce mot par « travail », en analysant de près la sémantique de ce mot. Pour les aspects «économiques » du travail, voir les études de P. Vidal-Naquet et M. Godelier.

4. Il faut signaler l'importance des recherches de Marcel Detienne et leur convergence avec celles de J.-P. Vernant. Pour certaines interprétations du monde grec on peut parler d'une approche J.-P. Vernant - M. Detienne. M. Detienne a élargi depuis lors ses travaux sur le plan méthodologique (1980) et sur les rapports entre l'écriture et le mythe (1989).

5. «En étudiant le travail, je ne pouvais pas ne pas m'apercevoir que du point de vue religieux, le travail agricole est inséré dans une conception où les divinités interviennent. L'acte de l'agriculteur est en même temps technique et religieux » (Vernant 1987 : 25). Toutefois cet aspect technique est à peine mentionné dans les pages auxquelles nous nous référons ici.

6. À propos du rapport entre la Cité naissante et le paysan hésiodien, voir B. Bravo (1985 : 721-732).

7. Il faut indiquer ici qu'en parlant de l'« artisanat », J.-P. Vernant limite son enquête dans Mythe et Pensée à ce type de métiers. La technè couvre, comme nous l'avons vu, un domaine qui dépasse ces activités humbles et inclut les « beaux-arts".

8. Voir l'ensemble des développements et des citations dans Mythe et Pensée: 208-211. En ce qui concerne Protagoras, ou plutôt le mythe de ce dernier exposé par Platon dans le dialogue qui porte le nom du sophiste, voir B. Cassin (1990) dont l'interprétation diverge de celle de J.-P. Vernant.

9. Il y aurait lieu dans ce domaine de tenir compte de l'incertitude qui ne peut pas être évacuée. Aristote souligne que «nous délibérons davantage sur les arts que sur les sciences, parce que nous sommes à leur sujet dans une plus grande incertitude ». À l'intérieur même du domaine des arts, on délibère davantage sur la navigation que sur la gymnastique étant donné « que la navigation a été étudiée d'une manière moins approfondie » (Éthique à Nicomaque III, 1112 b, 5 et suiv.).

10. En suivant une toute autre démarche, J.-M. Le Blond aboutit à des conclusions analogues: pour Aristote « le travail de l'artisan n'est pas essentiellement différent du travail de la nature et qu'une opération ait lieu dans 
des instruments artificiels ou dans des instruments naturels, cela ne fait pas la différence » (1970: 328 avec renvoi à la Génération des animaux IV, 767 a, 16). Voir toute la partie du livre en question consacré aux «schémas de l'industrie ».

11. Problème peut-être mal posé. La technologie moderne a montré que l'on n'a pas besoin de l'apport de la science pour construire une machine efficace - les premières machines à vapeur le montrent assez. Sur cet aspect de la question, voir les critiques de H. Joly (1974 : 216).

12. À cet égard, les études de J.-P. Vernant et M. Detienne constituent littéralement une « première » dans les études grecques et ouvrent un nouveau et vaste champ de recherche. À noter aussi une utilisation de la méthode structurale pleine de souplesse et une investigation poussée du champ sémantique de chaque terme. Le lecteur en est littéralement ébloui.

13. Voir par exemple Platon (Epinomis 976a-b). Aucune de ces activités ne peut atteindre la sagesse parce qu'elles sont fondées « sur les conjectures d'opinion » (doxais).

14. On attribué à Oppien les Cynégétiques qui sont également étudiées dans les Ruses de l'intelligence.

15. La tactique des Grecs à Salamine est comparée par Eschyle (Les Perses: 424-425) à la pêche aux thons. Voir à cet égard les développements de J.-P. Vernant et M. Detienne (R.I. : 280 et suiv).

\section{RÉFÉRENCES}

Bravo, B. 1985 Les Travaux et les jours et la cité, Annali della Scuola Normale Superiore di Pisa 15 : 707-765. Cassin, B. 1990 Le Lien rhétorique de Protagoras à Aelius Aristide, Philosophie 28 : 14-31.

Descat, R. 1986 L'Acte et l'effort : une idéologie du travail en Grèce ancienne (vilI ${ }^{e} v^{e}$ siècle av. J.-C.). Besançon, Paris: Les Belles Lettres.

Detienne, M. 1963 Crise agraire et attitude religieuse chez Hésiode. Bruxelles : Berchem (Latomus n 68).

- 1981 L'Invention de la mythologie. Paris : Gallimard.

- 1989 L'Écriture d'Orphée. Paris: Gallimard.

Detienne, M. \& Vernant J. -P. 1974 Les Ruses de l'intelligence. La mètis des Grecs. Paris : Flammarion.

Didonato, R. 1983 Postface. Une Euvre, un itinéraire. In L. Gernet, Les Grecs sans miracle. Paris : Maspéro (La découverte) : 403-420.

Finley, M. L. 1984 [1965] Innovation technique et progrès économique dans le monde ancien. In Économie et société en Grèce ancienne (Traduction française). Paris : La Découverte : 234-262.

— 1975 L'Économie antique (Traduction française). Paris : Éditions de Minuit.

Gernet, L. 1976 Anthropologie de la Grèce antique. Paris : Maspéro.

- 1983 Les Grecs sans miracle. Paris : Maspéro (La découverte).

Gille, B. 1980 Les Mécaniciens grecs. La naissance de la technologie. Paris : Seuil.

Humphreys, S.C. 1978 The Work of Louis Gernet. In Anthropology and the Greeks. London : Routlegde and Kegan Paul : 76-94.

Joly, H. 1974 Le Renversement platonicien. Paris : Vrin.

Le Blond, J.-M. 1970 Logique et méthode chez Aristote. Paris : Vrin.

Loraux, N. 1989 Ponos. Sur quelques difficultés de la peine comme nom du travail. In Les Expériences de Théresias. Paris: Gallimard : 54-76.

Malrieu, Ph. 1987 Dialectiques dans l'histoire des fonctions psychologiques. In Poikilia. Études offertes à Jean-Pierre Vernant. Paris : Éditions de l'EHESS : 437-450.

Meyerson, I. 1948 Les Fonctions psychologiques et l'ouvre. Paris : Vrin.

Vernant, J.-P. 1960 Sur les recherches de psychologie comparative historique, Journal de Psychologie 57 : 445-451.

- 1965 Mythe et pensée chez les Grecs. Études de psychologie historique. Paris : Maspéro.

— 1968a Préface. In L. Gernet 1968 : I-V.

- 1968b Introduction: In J.-P. Vernant (dir.) Problèmes de la guerre en Grèce ancienne. Paris : Mouton (republiée sous le titre La guerre des Cités. In Mythe et société en Grèce ancienne. Paris: Maspéro 1974) : 9-30. 
- 1979 À la table des hommes. Mythe de fondation du sacrifice chez Hésiode. In M. Detienne \& J.P. Vernant (dir.) La Cuisine du sacrifice en pays grec. Paris : Gallimard : 37-132.

- 1983 Préface. In L. Gernet Les Grecs sans miracle. Paris : Maspéro (La découverte) : 7-13.

- 1987 Entretien avec Jean-Pierre Vernant. Anthropologie historique et spécificité grecque, L'Ane 29: 25- 28.

\section{RÉSUMÉ}

Travail et technique chez les Grecs: l'approche de J.-P. Vernant. Analyse des travaux de J.-P. Vernant, ainsi que ceux de M. Detienne, se rapportant aux représentations de ce que l'on peut appeler travail et technique dans l'Antiquité grecque. En fait il n'y a pas de fonction du travail chez les Grecs. Au niveau de l'agriculture, le plan technique est difficilement distingué du plan théologique et rituel. Lartisan pour sa part reste enfermé dans sa propre « capacité ». Il réalise une « forme » qui se situe hors de lui sans pouvoir juger de l'efficacité de son œuvre : c'est l'usager qui est le meilleur juge de cette dernière. La rigidité de l'activité artisanale s'articule avec une conduite rusée - la mètis - qui, en contournant l'obstacle par tous les moyens, permet la réalisation du but recherché. Il est à noter que Detienne et Vernant sont les premiers à procéder à une analyse conséquente de la notion de mètis.

\section{ABSTRACT}

Work and technics in Ancient Greece ; J.-P. Vernant's approach. An analysis of the work of J. P. Vernant, as well as that of M. Detienne, concerning the representations of what might be called work and technics in Ancient Greece. In point of fact work as an independant function did not exist in Ancient Greece. In agriculture the technological aspect is hard to separate from the theological and ritual aspect. The artisan remains shut up in his own « capacity ». He renders concrete a « form » which exists separately from him without being able to judge whether his result is efficient or not: it is the consumer who is the best judge of efficiency. The rigor of artisanal activity is coupled with wisely behavior - the mètis - wich, by circumventing the barrier by all means available, allows reaching the desired goal. One must note that Vernant and Detienne are the first to analyze in a rational fashion the notion of mètis.

\section{NOTES ET RÉF́ERENCES DE L'ITRODUCTION}

1. Les références qui ne sont pas mentionnées sont fournies par Charles Kanelopoulos.

Photo d'ouverture « retour sur » : Jean-Pierre Vernant, sur le parvis du Collège de France improvisant un discours sur la recherche et les humanités avec les chercheurs du mouvement «Sauvons la Recherche » durant l'hiver 2004. Le Collège de France avait alors fermé ses portes au débat public et nous intervenions dans la rue face aux passants sur « l'utilité » et les raisons du métier de chercheur. Cliché F. Joulian, Paris 2004.

Bartholeyns, G. 2010 Le Paradoxe de l'ordinaire et l'anthropologie historique In E. Brilli, P.-O. Dittmar \& B. Dufal (dir.), Faire l'anthropologie historique du Moyen Âge. L'Atelier du Centre de recherches historiques, no $7<$ http://acrh.revues.org/index1928.html> 
Daumas, M. (dir.) 1962. Histoire générale des techniques, t. 1 Des Origines au XVé siècle. Paris: PUF.

Guillerme, J. \& Sebestik, J. 1966 et 2007 Les Commencements de la technologie, Thalès 12 : 1-72. Réédité dans Documents pour l'histoire des techniques $14: 49-122$.

Imbach, R. 2002 Die Arbor humanalis und die anthropologische relevanz der artes mechanicae In F. Domínguez Reboiras, P. Villalba Varneda \& P. Walter, Arbor scientiae. Turnhout : Brepols : 135-157.

Latour, B. 1987. Science in action: How to follow scientists and engineers through Society. Milton Keynes: Open University Press.

Latour, B. \& Woolgar, S. 1979 Laboratory life : the social construction of scientific facts. Beverly HillsLondres : Sage Publications.

Le Goff, J. 1971 Travail, techniques et artisans dans les systèmes de valeur du Haut Moyen Âge In Artigianato e technica nella società dell'alto Medioevo occidentale. Settimane di studio del centro itliano di studi sull'alto Medioevo XVIII. Spolète 1970 : 239-266.

Lévi-Strauss, C. 1962 La Pensée sauvage. Paris : Plon.

Mauss, M. 1967 (1947) Manuel d'ethnographie. Paris : Payot : 49-123.

— 1950 (1936) Les techniques du corps, Journal de psychologie 32 (3-4) : 365-372. Repris in Sociologie et anthropologie. Paris : PUF : 363-383.

Picon, A. 1992 Gestes ouvriers, opérations et processus techniques. La vision du travail des encyclopédistes, Recherches sur Diderot et sur l'Encyclopédie 13 : 131-147.

Schlanger, N. (éd.) 2006 Introduction à Marcel Mauss. Techniques, technology and civilisation. New York: Durkheim Press et Oxford: Berghahn Books.

Schmitt, J. -C. 1988 et 2001 Problèmes du mythe dans l'Occident médiéval, Razo. Cahiers du Centre d'études médiévales de Nice 8 : 3-17. Repris dans Le Corps, les rites, les rêves, le temps. Paris : Gallimard : 53-76.

- 1990. La Raison de gestes dans l'Occident médiéval. Paris : Gallimard.

Schwint, D. 2005 La routine dans le travail de l'artisan, Ethnologie française 3 (35) : 521-529.

- 2002 Savoir artisan de fabrication et détournement du temps, Sociétés 2 (76) : 33-48.

Simondon, G. 1956 Du mode d'existence des objets techniques. Paris : Aubier.

Sternagel, P. 1966 Die Artes mechanicae im Mittelalter. Begriffs- und Bedeutungsgeschichte bis zum Ende des 13. Jahrhunderts. Kallmünz : M. Lassleben.

Vernant, J. -P. 1952 Prométhée et la fonction technique, Journal de psychologie : 419-429. Repris dans Mythes et pensées chez les Grecs, cité par Kanelopoulos.

- 1955 Travail et nature dans la pensée ancienne, Journal de Psychologie: 1-29. Repris dans Mythes et pensées chez les Grecs, cité par Kanelopoulos.

- 1956 Aspects psychologiques du travail, La Pensée 66 : 80-84. Repris dans Mythes et pensées chez les Grecs, cité par Kanelopoulos.

- 1957 Remarques sur les formes et les limites de la pensée technique chez les Grecs, Revue d'histoire des sciences : 205-225. Repris dans Mythes et pensées chez les Grecs, cité par Kanelopoulos.

Vidal-Naquet, P. 1979 Étude d'une ambiguïté : les artisans dans la cité platonicienne In B. Vincent (éd.) Les Marginaux et les Exclus dans l'histoire, Cahiers Jussieu 5. Paris : UGE (10/18) : 232-261. Repris dans P. Vidal-Naquet Le Chasseur noir. Formes de pensée et formes de société dans le monde grec. Paris : Maspero (La Découverte), 1991 : 289-316.

Whitney, E. 1990 Paradise restored. The mechanical arts from antiquity through the thirteenth century, Philadelphia : The American Philosophical Society. 